Check for updates

Cite this: RSC Adv., 2019, 9, 17137

Received 18th March 2019

Accepted 23rd May 2019

DOI: $10.1039 / c 9 r a 02069 a$

rsc.li/rsc-advances

\section{Establishment and application of a dynamic tumor- vessel microsystem for studying different stages of tumor metastasis and evaluating anti-tumor drugs}

\author{
Bolin Jing, (D) ab Yong Luo, (D) ${ }^{c}$ Bingcheng Lin, ${ }^{d}$ Jianjun Li, ${ }^{\text {*a }}$ Zhuo A. Wang ${ }^{\text {*a }}$ \\ and Yuguang Du*a
}

Tumor metastasis is one of the main causes of cancer-related death, and it is difficult to study the whole process of tumor metastasis in vivo due to the complex physiological environment in the body. Therefore, it's crucial to develop simple and physiologically relevant in vitro cancer models to study the metastasis process, especially different phases of tumor metastasis. A novel microfluidic tumor-vessel co-culture system was established to reproduce the different phases of cancer metastasis (proliferation, migration, intravasation and adherence) individually in vitro for the first time. It was observed that blood vessels with fluid flow had big impact on metastasis of liver cancer cells HepG2 and breast ones MDAMB-231. In particular, it was found that both HepG2 and MDA-MB-231 cells migrated in the direction of "blood flow". Furthermore, MDA-MB-231 cells invaded through paracellular mode disrupting the intercellular endothelial junctions, whereas HepG2 cells engaged in transcellular intravasation through transcellular process. Compared with traditional assays, much more potent inhibition of 5-fluorouracil (5-Fu) on different phases of tumor metastasis was observed on the microsystem. In summary, the microfluidic device yielded abundant information about each phase of tumor metastasis, and would provide a powerful platform for use in drug screening, toxicology studies, and personalized medicine in future.

\section{Introduction}

Cancer is a serious human health problem worldwide. ${ }^{\mathbf{1}}$ It is well known that breast cancer is one of the leading causes of cancerrelated death among women in the world and liver cancer is one of the most lethal tumor diseases in China. ${ }^{2}$ However, metastasis remains one of the most poorly understood components of cancer pathogenesis. ${ }^{3}$ It has been proved that metastasis is responsible for as much as $90 \%$ of cancer-associated mortality. ${ }^{4}$ Anti-metastasis strategies are highly desired for curing cancer. To fully understand the metastasis process and the mechanism of malignant tumors, it is very crucial to monitor the metastasis process in real time and find corresponding ways to block cancer metastasis at the early stage.

It is well recognized that metastatic dissemination of cancer cells is a highly complex and multi-step biological process

${ }^{a}$ State Key Laboratory of Biochemical Engineering, Key Laboratory of Biopharmaceutical Production \& Formulation Engineering, PLA, Institute of Process Engineering, Chinese Academy of Sciences, Beijing 100190, P. R. China. E-mail: bljing@ipe.ac.cn; jjli@ipe.ac.cn; ygdu@ipe.ac.cn; Tel: +86-10-8254-5070

${ }^{b}$ University of Chinese Academy of Sciences, Beijing 100049, P. R. China

${ }^{c}$ School of Pharmaceutical Science and Technology, Dalian University of Technology, China

${ }^{d}$ Dalian Institute of Chemical Physics, Chinese Academy of Sciences, Dalian, China starting with invasion of cancer cells through the extracellular matrix (ECM) of the stroma toward the blood vessels. ${ }^{5}$ Metastasis of cancer cells is maintained by the dynamic interplay between tumor cells and many distinct types of cells that exist in the adjacent microenvironment, including endothelial cells, fibroblasts, and so on. ${ }^{6,7}$ The circulatory patterns and the microarchitecture of the capillary networks of the tumor microenvironment play key roles in the metastatic spread as well. ${ }^{8-10}$ Therefore, it is imperative to reconstitute a bionic tumor environment and establish a feasible and reliable evaluation system for cancer cell metastasis.

In vivo animal models are common platforms used to investigate tumor proliferation, but most of these models often suffered from the inability to directly observe and regulate the local microenvironment, ethical issues and high cost. Thus, the attempts to explore all phenomena related with tumor metastasis in vivo were greatly compromised. ${ }^{11,12}$ In this regard, in vitro models have been increasingly developed to allow for better control of the microenvironment and closer monitoring of the critical phenomena. ${ }^{\mathbf{1 3}, \mathbf{1 4}}$ For example, Truong and coworkers developed a 3D microfluidic co-culture system to investigate breast cancer cell invasion into a three dimensional tumor-stroma microenvironment. ${ }^{15}$ Sleeboom et al. found that MDA-MB-231 breast cancer cells and their cancer stem cells population migrated towards low oxygen in a microfluidic 
gradient device. ${ }^{\mathbf{1 6}}$ Zervantonakis et al. developed a microfluidicbased 3D model to recreate the tumor-vascular interface, allowing for studying tumor cell intravasation. ${ }^{17}$ So far, traditional in vitro models, which often studied only one phase of cancer metastasis, are unable to monitor each phase of tumor metastasis: proliferation, migration, and invasion, and lack of real-time information on invasion dynamics. ${ }^{18}$ Thus, it is critical to develop a more physiologically relevant in vitro cancer models to study and monitor the whole process and/or each phase of tumor metastasis and assess the effects of anti-tumor drugs on each phase of metastasis.

Here, a novel microfluidic-based tumor-vessel co-culture dynamic system was established to investigate the different phases of the cancer metastasis process and evaluate the effects of anti-cancer drug on each phase of tumor metastasis. A vascular cavity with fluid flow was built in the laminated microfluidic chip. The processes of proliferation, migration, intravasation and adherence of liver cancer cells HepG2 and breast ones MDA-MB-231 were studied within the device under fluid flow. Besides, all these processes in the dynamic tumorvessel microsystem were also compared with those under traditional static models. As a commonly used anti-tumor drug, the anti-metastatic effects of $5-\mathrm{Fu}$ on each phase of tumor metastasis were evaluated in the current microfluidic system.

\section{Materials and methods}

\subsection{Fabrication and assembly of the microfluidic device}

The tumor metastasis microsystem used in this study was fabricated from polydimethylsiloxane (PDMS). The PDMS plates, which were developed by Whitesides et al., were fabricated by soft lithography using photoresist (SU8 3035, Microchem) as the template. ${ }^{19}$ All microchannel layers were individually prepared by casting PDMS prepolymer $(10: 1 \mathrm{w} / \mathrm{w}$ ratio of PDMS to curing agent) on a microfabricated mold of the inverse channel design made of photoresist, and curing the polymer at $60{ }^{\circ} \mathrm{C}$ for $12 \mathrm{~h}$. After the microchannel layers was peeled from the wafer, peripheral holes $(1.5 \mathrm{~mm}$ diameter) for tubing were punctured on the PDMS plates. Additionally, $5 \mathrm{~mm}$ diameter holes were made in the center of each PDMS plate to facilitate connections between the tumor cells and the endothelial monolayer. Porous polycarbonate (PC) membranes with a $10 \mu \mathrm{m}$ pore size were placed between PDMS plates for on-chip cell culture. After careful alignment along the vertical direction, the PDMS plates were superimposed with the top and bottom PMMA frames, and fastened with screws.

\subsection{Culturing and tracking of cells}

Breast cancer cells MDA-MB-231 from ATCC were cultured in 1640 medium (1640, Gibco) supplemented with $10 \%(\mathrm{w} / \mathrm{v})$ of fetal bovine serum (FBS, Gibco). Liver cancer cells HepG2 were cultured in MEM medium containing $10 \%(\mathrm{w} / \mathrm{v})$ of fetal bovine serum and $1 \%(\mathrm{w} / \mathrm{v})$ of non-essential amino acids (NEAA, Gibco). Endothelial cells (EC) EAhy 926 were cultured in DMEM/ F12 medium (DMEM/F12, Gibco) containing 10\% (w/v) of fetal bovine serum. Penicillin (100 units per $\mathrm{mL}$, Gibco) and streptomycin (100 $\mu \mathrm{g} \mathrm{mL}{ }^{-1}$, Gibco) were added to all aforementioned media. All cells were cultured in a cell incubator with $5 \% \mathrm{CO}_{2}$ at $37{ }^{\circ} \mathrm{C}$.

Before the microdevice was assembled, all tubes and PDMS plates were pre-treated with $75 \%$ ethanol for $12 \mathrm{~h}$, and the entire system was then dried in oven at $60{ }^{\circ} \mathrm{C}$. The dried devices were exposed to ultraviolet light for $30 \mathrm{~min}$. The porous polycarbonate membrane was coated with collagen type I hydrogel at $37^{\circ} \mathrm{C}$. Human umbilical vein endothelial cells EAhy926 $(1 \times$ $10^{6}$ cells per $\left.\mathrm{mL}\right)$ stained by CellTracker ${ }^{\mathrm{TM}}$ Blue CMFDA $(10 \mathrm{mM}$, Invitrogen) were seeded on the porous polycarbonate membrane and incubated at $37{ }^{\circ} \mathrm{C}$ for 3-4 h, allowing seeded endothelial cells to attach to the membrane surface. Then, the microdevice was assembled as described above. The tubes were connected to the inlet and outlet holes at the top of the PMMA frame. The culture medium was infused into the inlets from the medium tanks with a multi-channel peristaltic pump (205 S/ CA12, WATSON MARLO) and flowed through the cavity between two layers of endothelial cells at a constant flow rate $\left(120 \mu \mathrm{L} \mathrm{h}^{-1}\right)$, which produces 1.6 dyn $\mathrm{cm}^{-2}$ shear stress within the normal physiological range. After two days of cultivation, tumor cells $\left(4 \times 10^{5}\right.$ cells per $\left.\mathrm{mL}\right)$, which were stained by CellTracker ${ }^{\mathrm{TM}}$ Green CMPTX ( $5 \mathrm{mM}$, Invitrogen) and wrapped by type I collagen gel, were seeded onto the upper endothelial monolayer, as shown in Fig. 1B.

\subsection{Analysis of cell viability on the chip}

The live/dead cell imaging kit (Molecular Probes, Life Technologies) was used to evaluate cell viability on the microfluidic device. In the live/dead cell assay, viable cells were stained green, while dead ones were stained red. Cells were loaded onto the microfluidic device. After several days of cultivation, the culture chambers on the microfluidic chip were flushed with phosphate buffered saline (PBS, HyClone) for 1-3 min. Cells were then incubated with the live/dead cell imaging reagents for 15-30 min at $37{ }^{\circ} \mathrm{C}$. Next, the culture chambers were flushed with PBS for 3-5 min to remove the reagents and observed under a fluorescent microscope. The percentage of cell viability was calculated by dividing the number of viable cells (green) by the total number of cells.

\subsection{Immunofluorescent staining}

This was done by following the standard protocol. EAhy 926 cells were fixed with $4 \%$ paraformaldehyde for $15 \mathrm{~min}$, and permeabilized with $0.1 \%$ Triton X-100 in PBS for $10 \mathrm{~min}$, and blocked with $3 \%$ BSA in PBS for $30 \mathrm{~min}$ at room temperature. Cells were then incubated with a VE-Cadherin Polyclonal Antibody (555661, BD Biosciences) at $10 \mu \mathrm{g} \mathrm{mL}^{-1}$ in blocking buffer for $1 \mathrm{~h}$ at room temperature, and washed with PBS, and incubated with a Goat anti-Rabbit IgG $(\mathrm{H}+\mathrm{L})$ Superclonal Secondary Antibody (A10474, Life Technologies) and Alexa Fluor® 594 conjugate at a dilution of $1: 1000$ for $1 \mathrm{~h}$ at room temperature. Cells were stained red, whereas nuclei were stained blue with DAPI (D1306, Invitrogen). Images were taken under a fluorescent microscope. 
A

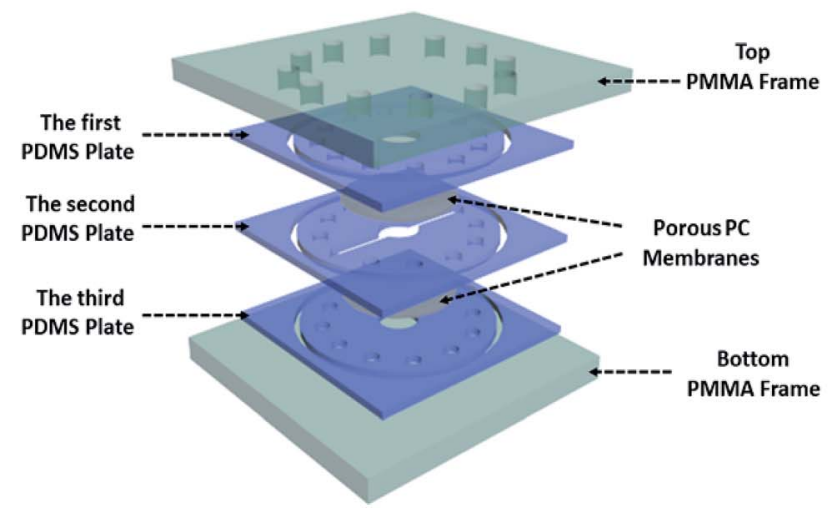

B

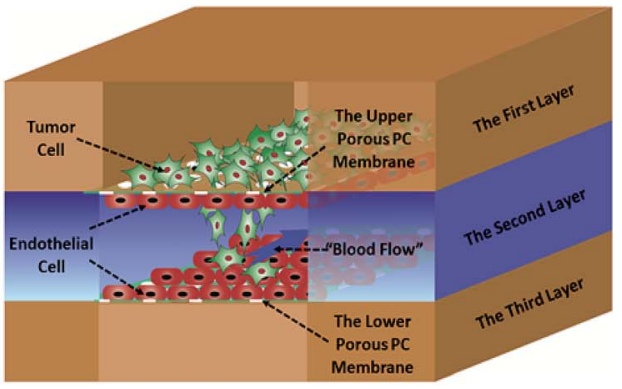

Fig. 1 The three-dimensional microsystem of tumor metastasis. (A) Annotated exploded view of the microfluidic device used in this study. (B) Conceptual diagram of the microsystem of tumor metastasis representing co-culture of tumor cells and the blood vessels.

\subsection{Functional characterization of the endothelial barrier}

The barrier-forming capacity of the endothelial monolayer formed by EAhy926 cells was evaluated by measuring the apparent permeability $\left(P_{\text {app }}\right)$ value of sodium fluorescein (Sigma) and FITC-labeled dextrans with different molecular weights (10 kDa, $40 \mathrm{kDa}$ and $70 \mathrm{kDa}$; Sigma) through the
A
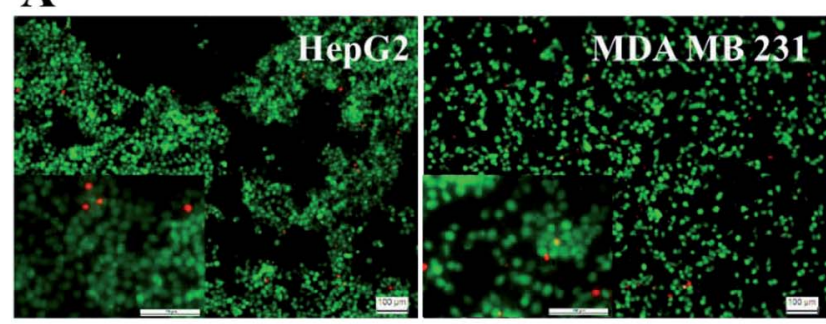

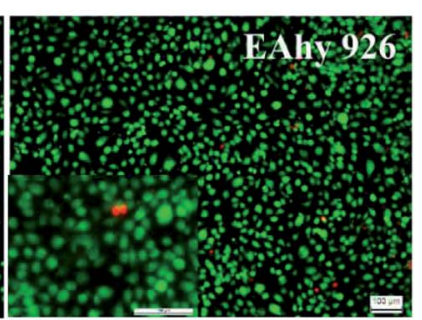

B

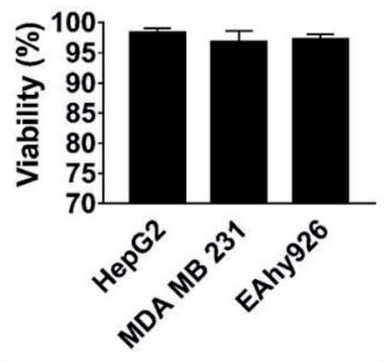

C

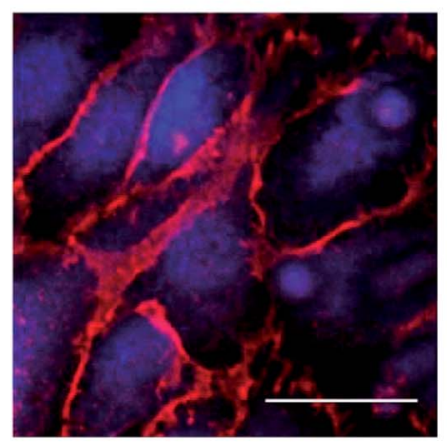

D

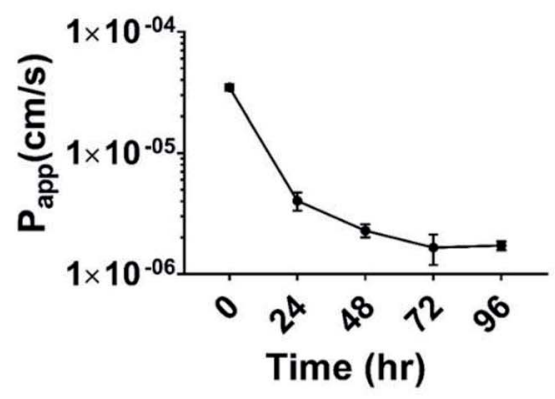

$\mathbf{E}$

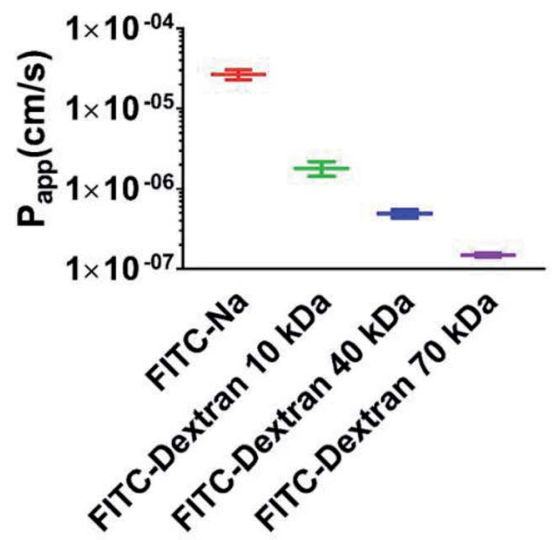

Fig. 2 In vitro co-culturing tumor cells and vascular endothelial cells on the microfluidics device. (A) Assessment of viability of HepG2, MDA-MB231 and EAhy 926 cells after $72 \mathrm{~h}$ cultivation on the device, and cells were stained using a live-dead kit and observed under a fluorescence microscope. Live cells appeared in green, whereas dead ones appeared in red (scale bar: $100 \mu \mathrm{m}$ ). (B) Viability analysis of HepG2, MDA-MB-231 and EAhy 926 cells. The error bars representing the standard deviations calculated from three parallel assays. (C) Confluency of the endothelial monolayer on the porous polycarbonate membrane coated with 3D ECM hydrogel stained with immunofluorescence (VE-cadherin, red) and DAPI (nuclei, blue) (scale bar: $50 \mu \mathrm{m})$. (D) Effects of incubation time on apparent permeability $\left(P_{\text {app }}\right)$ of FITC-dextran (10 kDa) across the endothelial monolayer. (E) The $P_{\text {app }}$ values of sodium fluorescein and FITC-dextrans with different molecular weight (10 kDa, $40 \mathrm{kDa}$ and $70 \mathrm{kDa}$ ) across the endothelial monolayer on the third day. 


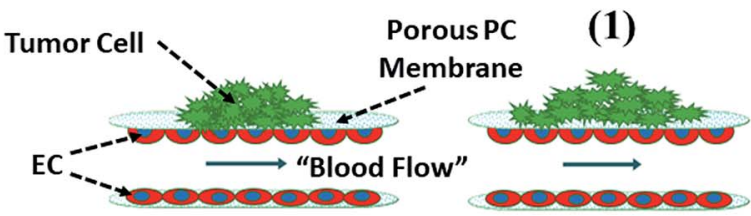

(2)

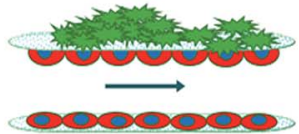

(3)

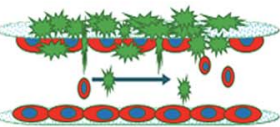

$0 \mathrm{hr}$

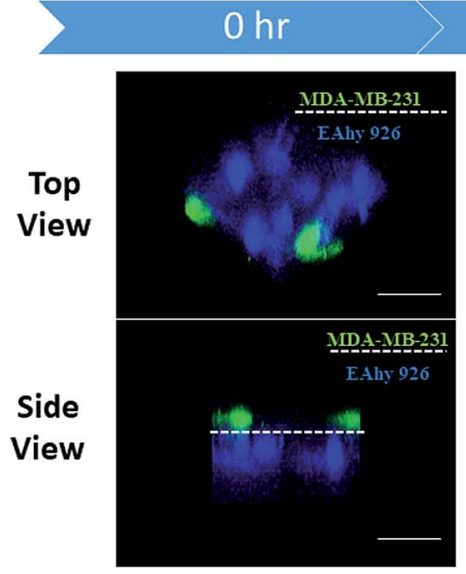

$6 \mathrm{hr}$

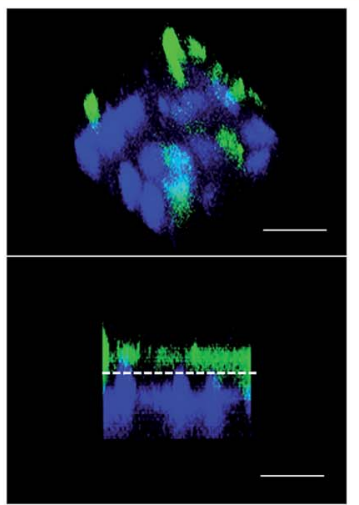

$18 \mathrm{hr}$

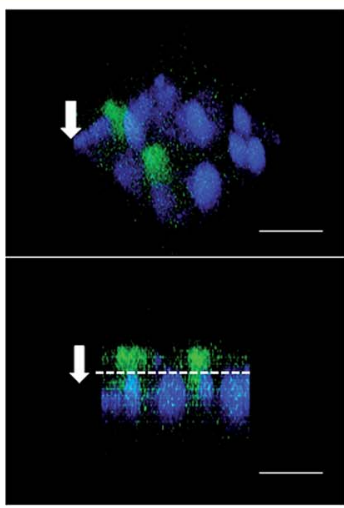

$24 \mathrm{hr}$

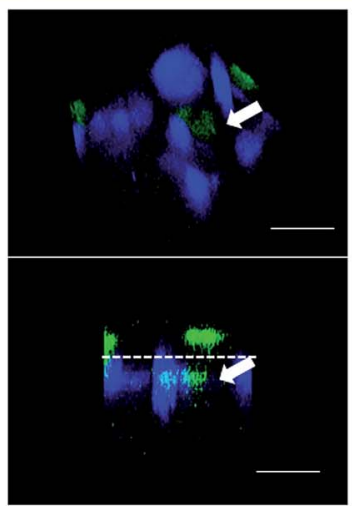

Fig. 3 Real-time monitoring the process of tumor cells metastasis: (1) liver cancer cells HepG2 (green) proliferated quickly after 6 h co-culturing with endothelial cells EAhy926 (blue); (2) tumor cells migrated through the extracellular matrix toward the endothelial monolayer from $6 \mathrm{~h}$ to $18 \mathrm{~h}$; (3) a small fraction of tumor cells in contact with the endothelial monolayer invaded into the "blood vessel lumen" (top and side views).

endothelial layer. The endothelial cell layer was clamped by two PDMS spacers. One milliliter of D'Hanks solution containing sodium fluorescein $\left(2 \mathrm{nmol} \mathrm{mL}^{-1}\right)$ or FITC-dextrans $(2 \mathrm{nmol}$ $\mathrm{mL}^{-1}$ ) with different molecular weights was perfused through the microchannel of the upper PDMS layer, and the blank D'Hanks solution was circulated through the lower microchannel at a flow rate of $120 \mu \mathrm{L} \mathrm{h}^{-1}$. $P_{\text {app }}$ was calculated using the equation below: ${ }^{20}$

$$
P_{\text {app }}\left[\mathrm{cm} \mathrm{s}^{-1}\right]=\left(1 / A C_{0}\right)(\mathrm{d} Q / \mathrm{d} t)
$$

where $A=$ area of mass transfer, $C_{0}=$ donor concentration of reagent in the upper medium, and $\mathrm{d} Q / \mathrm{d} t=$ transmembrane transportation rate.

\subsection{Evaluation of anti-metastatic effect of 5-fluorouracil (5- Fu)}

After the cancer model was established on the chip as above, the medium which was infused into cavity between two layers of endothelial cells was replaced with the fresh culture medium containing 5-Fu at concentrations of $0.1 \mu \mathrm{g} \mathrm{mL}{ }^{-1}, 1 \mu \mathrm{g} \mathrm{mL} L^{-1}, 10$ $\mu \mathrm{g} \mathrm{mL}^{-1}$, and $100 \mu \mathrm{g} \mathrm{mL^{-1 }}$ respectively. After $24 \mathrm{~h}$ cultivation, the numbers of tumor cells that proliferated, migrated and intravasated were counted at $20 \times$ magnification under a light microscope individually. Each type of cells was assayed in quintuplicate and repeated at least twice.

\subsection{MTT assay}

For static proliferation assay, MTT assay was done. Tumor cells were seeded in 96-well plates at a density of $5 \times 10^{3}$ cells per well. After $24 \mathrm{~h}$ cultivation, cells were incubated in the absence or presence of various concentrations of 5-Fu for another $24 \mathrm{~h}$. Then, MTT solution ( $5 \mathrm{mg} \mathrm{mL}$ ) was added onto the plates, and incubated at $37{ }^{\circ} \mathrm{C}$ for $4 \mathrm{~h}$. Formazan, derived from MTT by living cells, was dissolved in DMSO $(150 \mu \mathrm{L}$ per well), and the absorbance at $570 \mathrm{~nm}$ was measured. All MTT experiments were performed in octuplicate and repeated at least 3 times.

\subsection{Wound healing assay}

For static migration assay, wound-healing assay was performed. Tumor cells $\left(1 \times 10^{5}\right.$ cells per $\left.\mathrm{mL}\right)$ stained by CellTracker ${ }^{\mathrm{TM}}$ Green CMPTX (5 mM) were seeded in 24-well dishes and incubated for $24 \mathrm{~h}$, monolayers were then disrupted with a cell scraper ( $1 \mathrm{~mm}$ wide). After $18 \mathrm{~h}$ incubation with or without various concentrations of $5-\mathrm{Fu}$, photographs were taken under a fluorescence microscope to calculate the number and migration distance of tumor cells. Experiments were carried out in triplicate, and three fields were recorded in each well.

\subsection{Transwell assay}

For static invasion assays, $5 \times 10^{4}$ cells per $\mathrm{mL}$ tumor cells stained by CellTracker ${ }^{\mathrm{TM}}$ Green CMPTX $(5 \mathrm{mM})$ were plated in the top chamber with collagen type I hydrogel coated membrane (24-well insert, pore size: $10 \mathrm{~mm}$ ). Serum-free medium was added into the upper chamber, and the medium containing $20 \%$ FBS was added into the lower chamber as a chemoattractant. After $24 \mathrm{~h}$ incubation in the absence or presence of various concentrations of $5-\mathrm{Fu}$ at $37{ }^{\circ} \mathrm{C}$, cells that invaded through the pores into the lower surface of the membrane were counted under a fluorescence microscope. Three invasion chambers were used per condition, and five fields were recorded for each well. 


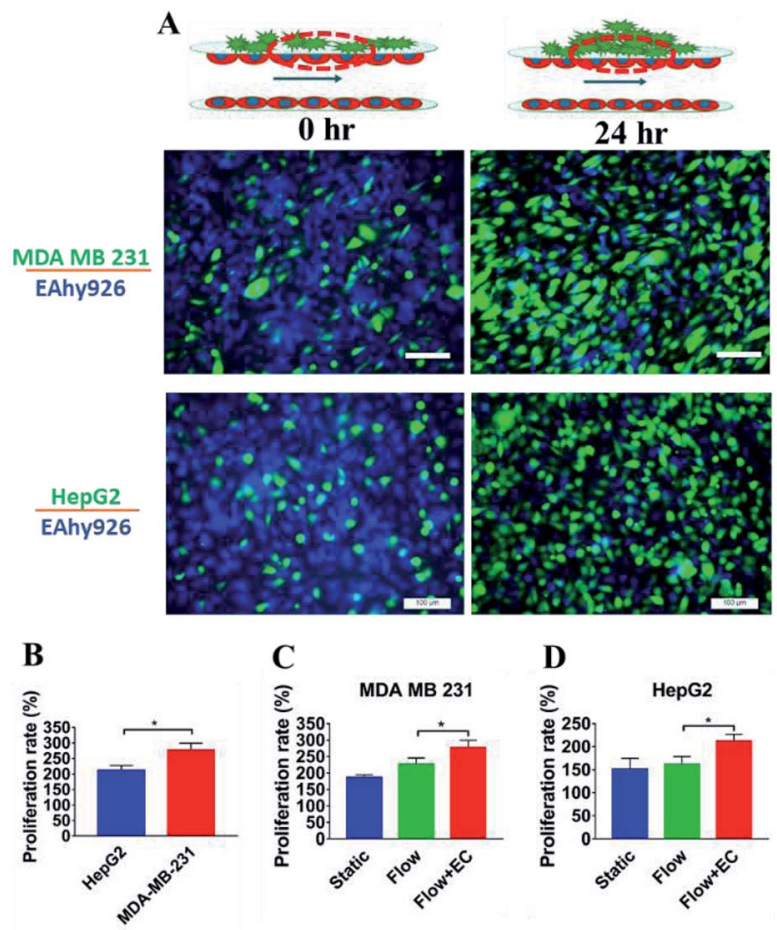

Fig. 4 Impact of the endothelium layer on proliferation of tumor cells. (A) Schematic views of proliferation of tumor cells on the device and confocal images of tumor cells (green) on the endothelial monolayer (blue) at $0 \mathrm{~h}$ and $24 \mathrm{~h}$ (scale bar: $100 \mu \mathrm{m}$ ). (B) Proliferation rates of MDA-MB-231 and HepG2 cells on the microfluidic system in the presence of endothelium during $24 \mathrm{~h}$. (C) Proliferation rates of MDAMB-231 under static conditions (Static) and on the microfluidic device in the absence (Flow) and presence (Flow + EC) of endothelium (EC) monolayer during $24 \mathrm{~h}$. (D) Proliferation rates of HepG2 under static conditions (Static) and on the microfluidic device in the absence (Flow) and presence (Flow + EC) of endothelium monolayer during $24 \mathrm{~h}$.

\subsection{Data analysis and quantification}

All data were analyzed by averaging the values of at least three microfluidic devices, with each device representing one independent experiment. The mean value of each device was calculated based on the average of at least 5 hydrogel ECM regions, equivalent to selecting at least 50 tumor cells per device. Student's tests (two-sample, $P<0.05$ ) and correlation analysis were performed with GraphPad Prism.

\section{Results and discussion}

\subsection{Microfabrication and evaluation of the microfluidic device}

This laminated microfluidic device includes two pieces of polymethylmethacrylate (PMMA) frames and three layers of PDMS plates with a diameter of $30 \mathrm{~mm}$ and thicknesses of $2 \mathrm{~mm}$, which sandwich two layers of porous polycarbonate (PC) membranes with a $10 \mu \mathrm{m}$ of pore size (Fig. 1A). Microchannels (0.2 mm deep, $0.8 \mathrm{~mm}$ wide, and $18 \mathrm{~mm}$ long) were fabricated on each PDMS plate for perfusing cell culture media. As shown in Fig. 1B, on the inside of two layers of porous PC membranes, endothelial cells were seeded and a hollow vascular lumen was formed, tumor cells packaged by collagen type I were seeded on the surface of endothelial monolayer on the first layer of the microfluidic system.

Viability of liver cancer cells HepG2, breast ones MDA MB 231, and vascular endothelial cells EAhy926 was evaluated after they were cultivated on the microsystem for $72 \mathrm{~h}$. PDMS has been demonstrated to be a biocompatible material with a vast array of cell types. ${ }^{21}$ Consistent with previous studies, ${ }^{22}$ our results also confirmed that cancer and endothelial cells showed around $97 \%$ viability on the microdevice after 3 days of cultivation (Fig. 2A and $\mathrm{B}$ ), indicating that the laminated PDMS microfluidic devices almost had no detrimental effects on cell survival.

To function as the endothelial barrier, EAhy926 cells were cultured on the porous polycarbonate membrane for 3 days on the microdevice to form tight junctions. The endothelial cellcell junctions were visualized by immunofluorescent staining VE-cadherin of EAhy 926 cells using a vascular endothelialcadherin (VE-cadherin) antibody. As shown in Fig. 2C, endothelial cells formed the continuous cell-cell junctions spanning the whole area of the porous PC membrane. Furthermore, the barrier function of the HUVEC layer was assessed by measuring the $P_{\text {app }}$ values of sodium fluorescein and FITC-labeled dextrans with different molecular weights $(10 \mathrm{kDa}, 40 \mathrm{kDa}$ and $70 \mathrm{kDa})$ across the endothelial layer. $P_{\text {app }}$ of FITC-dextran $(10 \mathrm{kDa})$ was reduced gradually with extension of incubation time, and was stable at about $1.5 \times 10^{-6} \mathrm{~cm} \mathrm{~s}^{-1}$ after 3 days of cultivation (Fig. 2D), proving that the endothelial layer became fully dense on the third day. In addition, the $P_{\text {app }}$ values of FITC-dextran (70 $\mathrm{kDa})$ and FITC-dextran $(40 \mathrm{kDa})$ reached $1.5 \times 10^{-7}$ and $4.9 \times$ $10^{-7} \mathrm{~cm} \mathrm{~s}^{-1}$ on the third day, were lower than that of FITCdextran $(10 \mathrm{kDa})$, suggesting that $P_{\text {app }}$ was inversely correlated with the molecular weights of FITC-dextran (Fig. 2E). These data demonstrated that the endothelial monolayer on the microfluidic device formed a size-selective barrier for trans-endothelial transport, which was similar to those on other microfluidic systems and engineered blood vessels on 3D matrices. ${ }^{23,24}$

The innovative aspect of our microsystem was to co-culture tumor cells packaged by collagen type I with endothelial cells under constant fluid flow to mimic the environment of tumor metastasis in vivo. Blood vessel is a crucial biophysical aspect of the tumor microenvironment, and has been heavily studied in metastasis of tumor cells. ${ }^{25,26}$ However, only several studies focused on co-culturing tumor cells with endothelial cells on a two-dimensional plates rather than seeding tumor cells on the surface of endovascular cortex, which may not accurately represent physiological behavior of tumor cells. ${ }^{27,28}$

\subsection{Evaluation of tumor metastasis on the microsystem}

When serum-free culture medium was flowed through the first layer of the microsystem and the medium containing $20 \%$ of fetal bovine serum was infused into the inlets of the second layer between endothelial cells, the whole process of tumor metastasis was directly observed on the device during $24 \mathrm{~h}$, as shown in Fig. 3. A number of invasion models of co-culturing cells that mimiced the tumor microenvironment using 
A

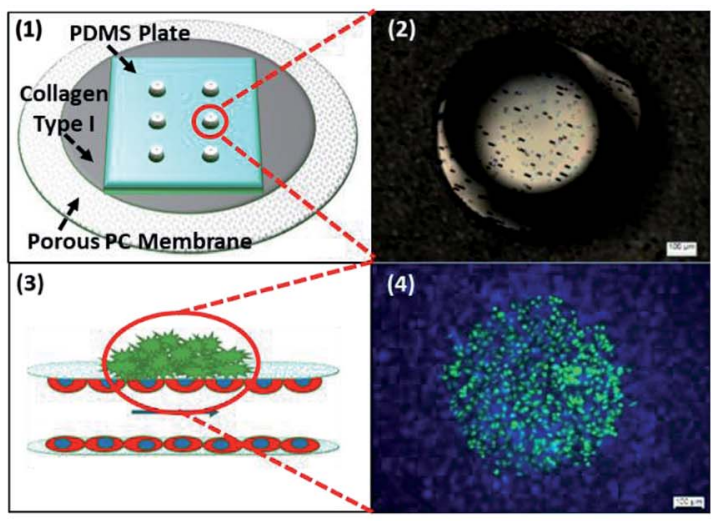

C

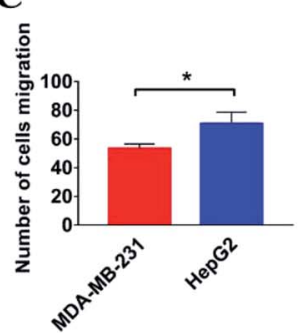

D

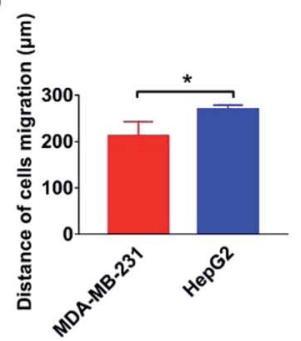

B

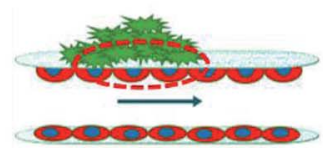

$0 \mathrm{hr}$

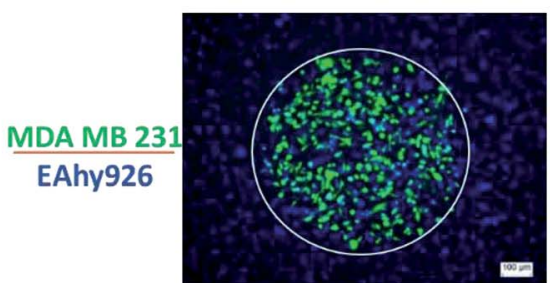

HepG2

EAhy926

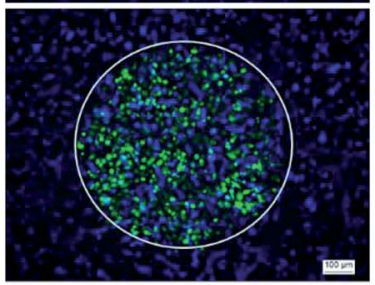

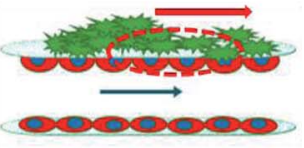

$18 \mathrm{hr}$

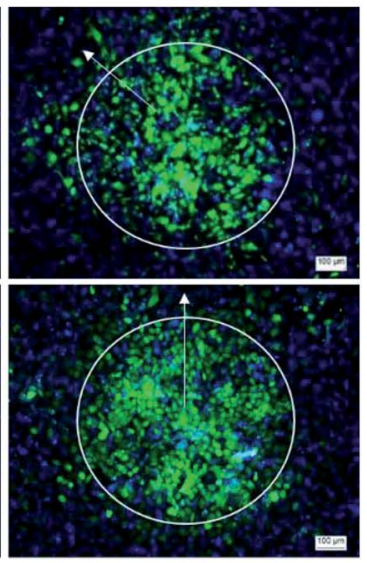

Fig. 5 Effects of "blood flow" between two endothelial monolayers on tumor cells migration. (A) Fabrication of 3D tumor cell clusters: (1) six holes with a diameter of $0.5 \mathrm{~mm}$ punched on each PDMS plate, which was put on the porous polycarbonate membrane coated with collagen type I hydrogel; (2) magnification of one hole with the magnifying power of $100 \times$ (scale bar: $100 \mu \mathrm{m}$ ); (3) schematic view of a 3D tumor cell cluster on the endothelial monolayer; (4) confocal image of a 3D tumor cell cluster on the endothelial monolayer (scale bar: 100 $\mu$ m). (B) Schematic views of the device showing migration of tumor cells on the endothelial monolayer (top) and confocal images demonstrating migration of tumor cells (MDA MB 231 and HepG2, green) on the endothelium monolayer (EAhy926, blue) (scale bar: 100 $\mu$ m) (bottom). (C) The numbers of tumor cells migrating into the extracellular matrix after $18 \mathrm{~h}$. (D) Comparison of the migration distance of tumor cells under static conditions (Static) and on the microfluidic device in the presence of endothelium (EC) monolayer (EC + Flow) after18 $\mathrm{h}$.

microfluidic devices have been reported. ${ }^{29,30}$ However, thus far most microfluidic-based models mainly focused on one step of cancer metastasis such as migration. Inspired by these results, different stages of tumor metastasis were further investigated on the microsystem.

\subsection{Proliferation and migration of tumor cells on the endothelial monolayer on the microsystem}

Proliferation of tumor cells is the first step of tumor metastasis. $^{31}$ To establish a physiologically relevant proliferation model for tumor cells, human cancer cells were seeded on the endothelial monolayer within the microsystem, and the culture medium was flowed across two endothelial cell layers providing nutrients for tumor cells. After $24 \mathrm{~h}$ of co-culturing, the fluorescence images of cancer cells' nuclei demonstrated that significant proliferation was observed in a number of tumor cells (Fig. 4A) $(P=0.0026)$. Under the conditions of the same cell seeding density and $24 \mathrm{~h}$ of cultivation, the proliferation rates of MDA-MB-231 and HepG2cells reached 277\% and 212\% respectively, indicating that the proliferative capacity of MDAMB-231 cells was higher than that of HepG2 cells on the microdevice (Fig. 4B). For comparison, two types of tumor cells were also cultured on the microfluidic system in the absence of the endothelial monolayer and on 96-well plate for $24 \mathrm{~h}$.
Quantitative analyses showed that the proliferation rates of two types of tumor cells on our microfluidic system in the presence of the endothelial monolayer were higher than in the absence of endothelial monolayer respectively (Fig. 4C and D). Thus, it was confirmed that the proliferative capability of tumor cells cultured on the microfluidic system in the presence of the endothelial monolayer was significantly higher than that of those cultivated in the absence of the endothelial monolayer. Similar results were reported previously, since new blood vessels would be formed to provide nutrition and oxygen for tumor proliferation at the early stage of tumor metastasis. ${ }^{\mathbf{8}, 32}$

After tumor cells proliferated to a certain number, they exhibited migration characteristics across the extracellular matrix toward the endothelial monolayer. ${ }^{33}$ In order to build a tumor migration model close to the physiological environment in vivo, six tumor cell clusters packaged by $3 \mathrm{mg} \mathrm{mL}^{-1}$ of collagen type I with a diameter of $0.5 \mathrm{~mm}$ were seeded on the endothelial monolayer within the microsystem (Fig. 5A). After $6 \mathrm{~h}$, tumor cells adopted heterogeneous morphology with round and elongated shapes, and started migrating toward the outer regions of seeded cancer cells by 18 h (Fig. 5B). Notably, MDAMB-231 and HepG2 cells exhibited different migration characteristics. According to the statistical analysis of the distance and number of tumor cells that migrated, it was concluded that the 
A

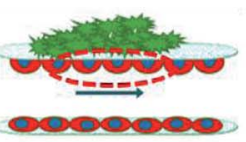

0 hr
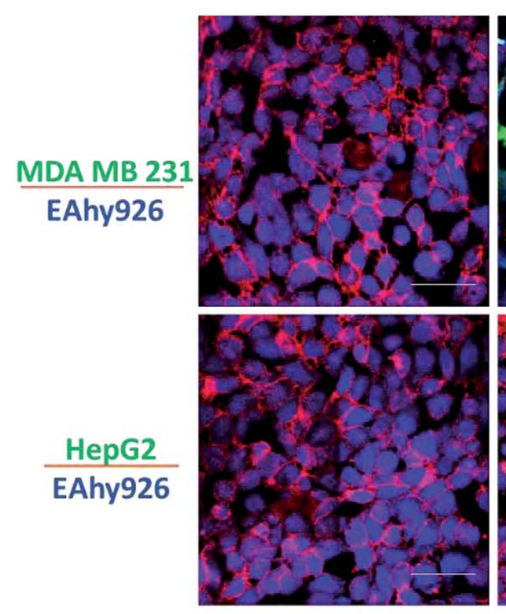

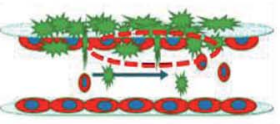

$24 \mathrm{hr}$
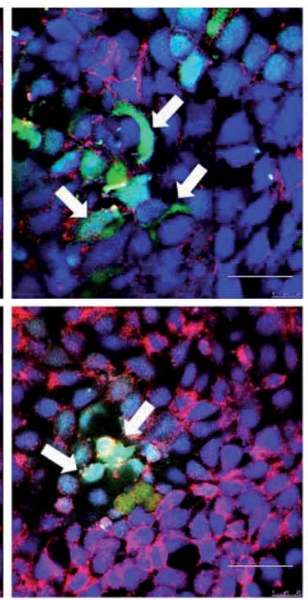

B

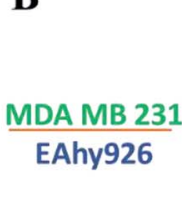

HepG2
EAhy926

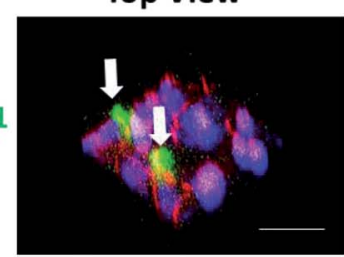

Top View

Side View

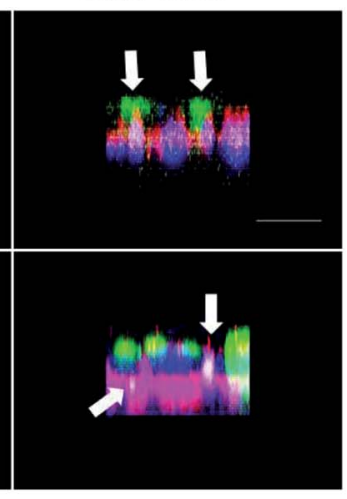

C

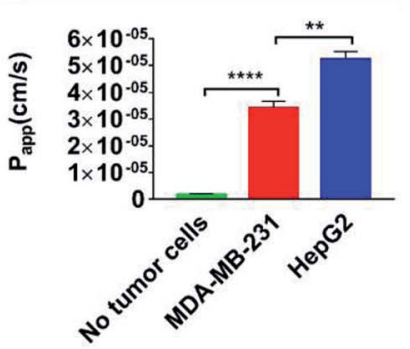

D

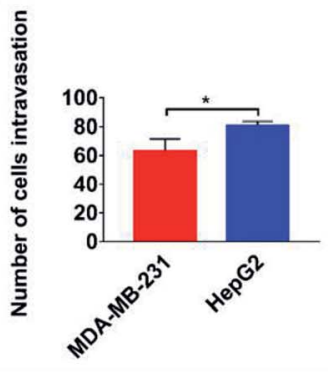

Fig. 6 Intravasation of tumor cells. (A) Schematic views of the device containing tumor cells invading into the endothelial monolayer (top), and confocal images of intravasation of tumor cells (MDA-MB-231 and HepG2, green) across the endothelium monolayer (EAhy926, blue) destroying the intercellular protein (VE-cadherin, red) of endothelial cells on the microfluidic system (bottom) after $24 \mathrm{~h}$ (scale bar: $50 \mu \mathrm{m}$ ). (B) Confocal images showing different intravasation routes of tumor cells (MDA-MB-231 and HepG2): MDA-MB-231 invaded through paracellular mode of intravasation disrupting the intercellular endothelial junctions, whereas HepG2 engaged in transcellular intravasation and entered mainly through the endothelial cell body (scale bar: $50 \mu \mathrm{m}$ ). (C) Effects of intravasation of tumor cells (MDA-MB-231 and HepG2) on $P_{\text {app }}$ of FITC-dextran (10 kDa) across the endothelial monolayer on the microfluidic device after $24 \mathrm{~h}$. (D) Comparison of the number of tumor cells (MDA-MB-231 and HepG2) invading into the blood vessel lumen on the microfluidic device after $24 \mathrm{~h}$ cultivation.

migration ability of HepG2 cells was significantly stronger than that of MDA-MB-231cells (Fig. 5C and D) $(P=0.0255)$. It was also found that the migration direction of tumor cells was consistent with the direction of "blood flow", an interesting discovery on this in vitro model (Fig. 5B). Other findings demonstrated that interstitial flow dramatically increased the motility of tumor cells, and tumor cells migrated in the direction of the flow. ${ }^{34,35}$ In particular, autologous chemotaxis, a directed cell migration induced by blood flow, has been identified as a potentially important mechanism of cancer migration. ${ }^{36,37}$ Thus, these results indicated that the blood vessels with fluid flow may play critical roles in controlling the migration direction of tumor cells.

\subsection{Intravasation and adherence of different tumor cells onto endothelial monolayer on the microsystem}

Intravasation of tumor cells into blood vessels was an important process of tumor metastasis, which was characterized by migration of tumor cells from the 3D matrix across the basal endothelial surface and subsequent appearance on the apical endothelial surface inside the vascular lumen. ${ }^{38}$ Fig. 6A demonstrated the characteristics of the intravasation process, in which cancer cells first adhered to the apical surface of the endothelium, then destroyed the intercellular protein of endothelial cells, and finally invaded into the "blood vessel lumen". Due to heterogeneous morphology of different types of tumor cells, the structures of tumor cells were labeled to further investigate their invasion behavior. Interestingly, it was observed that there was a substantial difference between the intravasation phenotypes of MDA-MB-231 and HepG2 cells. Intercellular proteins (VE-cadherin) of endothelial cells were destroyed seriously by MDA-MB-231 cells, invading into the "blood vessel lumen" mainly through intercellular space of the endothelial monolayer. However, HepG2 cells invaded into the endothelial monolayer mainly through the EC body, disrupting the surrounding basement membrane (Fig. 6A and B).

These results suggested that the routes of transendothelial migration may be related with the cancer cell types. Several previous studies reported that tumors cells can intravasate into the circulating stream in a paracellular fashion by remodeling cell-cell junctions on the lining of the vessel. ${ }^{39,40}$ Additionally, cancer cells can engage in transcellular migration (TCM) where they cross the endothelial cell cytoskeleton and membrane by creating transitory pore-like structures. ${ }^{13}$ Most cancer cells seem to use a paracellular mode of transendothelial migration in vitro, and there were only a few examples about the transcellular route. ${ }^{41}$ Due to the complex physiological environment in the 
A

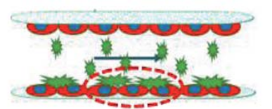

body, it is not clear which route is actually used by cancer cells in vivo. ${ }^{42}$

Intravasation was a rare and inefficient process, and occurred only for a small fraction of tumor cells in contact with the endothelial monolayer. ${ }^{43}$ To examine the detrimental effects of tumor cells invasion on the endothelial monolayer, the changes of the endothelial barrier were monitored. A statistically significant 10 -fold increase in endothelial $P_{\text {app }}$ of FITCdextran (10 kDa) was observed (Fig. 6C) $(P<0.0001)$. To compare the invasive ability of MDA-MB-231 and HepG2 cells, the changes of endothelial $P_{\text {app }}$ of FITC-dextran $(10 \mathrm{kDa})$ and the number of tumor cells invading into the "blood vessel lumen" were recorded and calculated (Fig. 6C and D). According to the statistical analysis, the number of HepG2 cells invading into "blood vessel lumen" was around 79 cells per $\mathrm{mm}^{2}$, while the number of MDA-MB-231 cells reached 64 cells per $\mathrm{mm}^{2}$. And the increase in endothelial $P_{\text {app }}$ of FITC-dextran $(10 \mathrm{kDa})$ after intravasation of HepG2 cells was much higher than that after intravasation of MDA-MB-231 cells. These results demonstrated that the invasive capacity of HepG2 cells was stronger than that of MDA-MB-231 cells. This phenomenon may be related with the different routes of transendothelial migration of two tumor cells: MDA-MB-231 cells invaded through paracellular mode disrupting the intercellular endothelial junctions, whereas HepG2 cells engaged in transcellular intravasation through destroying the vascular endothelium.

Trafficking of tumor cells through the vasculature and the arrest of circulating tumor cells on the endothelial layer at

\section{A Proliferation}

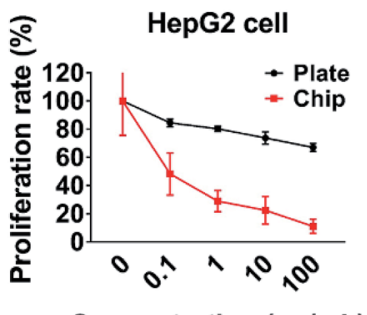

Concentration $(\mu \mathrm{g} / \mathrm{mL})$

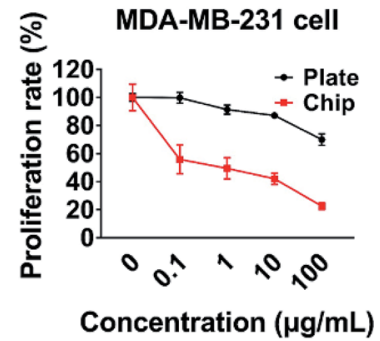

B Migration
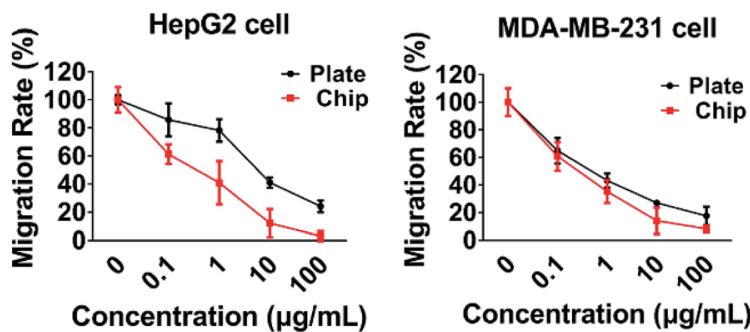

C Invasion/Intravasation
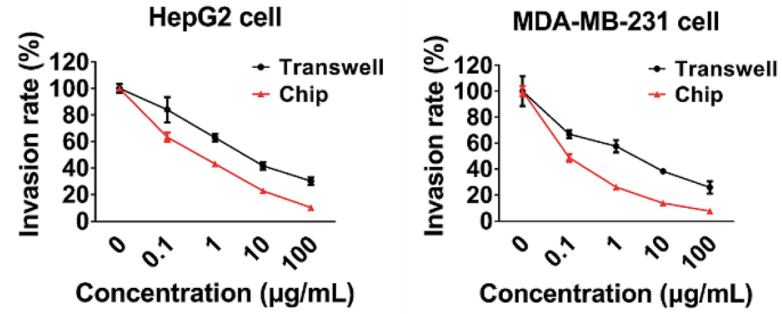

Fig. 8 Comparison of the rates of proliferation (A), migration (B) and invasion/intravasation (C) of cancer cells in the presence of different concentrations of 5-fluorouracil on traditional 2D platform and our 3D microfluidic device.

specific sites are critical steps for metastatic cancer. ${ }^{\mathbf{4 4}}$ The current microfluidic device was run at defined flow rates within the range of physiologic levels between two layers of endothelial cells, and a part of tumor cells, invading into the "blood vessel lumen", adhered onto the surface of the lower layer of endothelial cells. As demonstrated by the fluorescence images of cells' nuclei, tumor cells were successfully arrested on the endothelial layer (Fig. 6A). The diameter of tumor cells was longer than $25 \mu \mathrm{m}$, which was longer than the suspended ones $(10 \mu \mathrm{m})$, demonstrating that tumor cells adhered onto the surface of endothelial cells (Fig. 7B). And it was also found that a significantly higher number of HepG2 cells ( 51 cells per $\mathrm{mm}^{2}$ ) adhered onto the surface of endothelial monolayer in comparison with MDAMB-231 cells (32 cells per $\left.\mathrm{mm}^{2}\right)$ (Fig. 7C) $(P=$ 0.003 ). Therefore, this microdevice could be potentially used for identification of the molecular determinants of trafficking and the arrest of circulating cancer cells on endothelium at specific sites of metastatic cancer, which has been limited in large part by challenges in studying the intravascular microenvironment under physiological conditions. ${ }^{11}$ 


\subsection{Evaluation of the anti-metastatic effects of 5-Fu on the} microdevice

5-Fluorouracil (5-Fu) has been widely used for more than 30 years and is still considered to be one of the key drugs for treatment of cancers of liver, breast, ovary, and gastrointestinal tract. ${ }^{4,46}$ To set up a platform for assessing and comparing the effects of anti-metastatic drug on different phases of tumor metastasis, the current microfluidic system was used to evaluate the anti-metastatic effects of 5-Fu. Firstly, as shown in Fig. 8A, compared with the traditional $2 \mathrm{D}$ cell culture plates, the proliferation rates of both HepG2 and MDAMB-231 cells that were treated with the same concentrations of $5-\mathrm{Fu}$ on the microsystem were greatly reduced. And the inhibitory effect of 5-Fu on the proliferation rate of HepG2 cells was more potent than that of breast cancer cells MDAMB-231 on the microfluidic device. When HepG2 cells were treated with 5 -Fu at $0.1 \mu \mathrm{g} \mathrm{mL}{ }^{-1}$, the proliferation rate of HepG 2 was reduced by $52 \%$ relative to the untreated control. Secondly, the migration abilities of both MDAMB-231 and HepG2 cells were seriously inhibited by 5-Fu both on the chip and plate, and the inhibitory effects of 5-Fu at $100 \mu \mathrm{g} \mathrm{mL}^{-1}$ on the migration rate of both HepG2 and MDAMB231 on our device and plate were above $80 \%$ (Fig. 8B). Finally, the intravasation rate of MDAMB-231 cells was more strongly inhibited by $5-\mathrm{Fu}$ than that of HepG2 cells on the microfluidic system, and inhibition of $5-\mathrm{Fu}$ at $1 \mu \mathrm{g} \mathrm{mL} \mathrm{mL}^{-1}$ on intravasation of MDAMB-231 cells reached 73\% (Fig. 8C). However, the inhibition rate of $5-\mathrm{Fu}$ on the invasion of HepG2 cells was similar to MDAMB-231 cells in the Transewell (Fig. 8C). Besides, the inhibitory effects of $5-\mathrm{Fu}$ on proliferation, migration, and intravasation of HepG2 and MDAMB-231 cells were all positively related to the concentrations of $5-\mathrm{Fu}$.

These results suggested that endothelial cells with "blood flow" may be critical for the efficacy of 5-Fu on cancer metastasis. During the proliferative phase of tumor cells, functional vasculature and interstitial fluid flow may have major influence on drug transport. ${ }^{22}$ At the stage of tumor cell intravasation, due to the barrier function of vascular endothelial cells ${ }^{28}$ and the different invasion style of various tumor cells, ${ }^{\mathbf{4 1}}$ the inhibitory effects of drugs on tumor cells intravasation may be different. Therefore, tumor-vascular models such as our dynamic microfluidic platform may become capable of recapitulating the desired, human-mimicking, and nuanced responses to drug treatment at different phases of tumor metastasis.

\section{Conclusions}

In summary, a dynamic co-culture microfluidic system was established to mimic the whole process of cancer metastasis and assess the effects of anti-cancer drug on different phases of metastasis. For the first time, it was found that proliferation, migration, and intravasation of tumor cells were significant influenced by endothelial cells with "blood flow" in vitro, and much more significant inhibition effects of $5-\mathrm{Fu}$ on proliferation, migration and intravasation of cancer cells were observed than those on traditional static models due to the possible influence of blood vessel on drug transport, and MDA-MB-231 and HepG2 cells invaded through paracellular mode and transcellular one respectively. This microfluidic system provided a better way to mimic the in vivo tumor microenvironment, enabling further exploration of cancer metastasis and evaluation of anti-tumor metastasis drugs for different phases of metastasis. Ultimately, this powerful platform will be potentially used in future drug evaluation, toxicology studies, and personalized medicine.

\section{Conflicts of interest}

There are no conflicts to declare.

\section{Acknowledgements}

We would like to thank Dr Chuanfang Chen from the Public Technology Service Center of Institute of Electrical Engineering, Chinese Academy of Sciences, for his assistance with the laser confocal photography. This work was financially supported by the National Natural Science Foundation of China (No. 31870802).

\section{Notes and references}

1 J. Ferlay, I. Soerjomataram, R. Dikshit, S. Eser, C. Mathers, M. Rebelo, D. M. Parkin, D. Forman and F. Bray, Cancer incidence and mortality worldwide: sources, methods and major patterns in GLOBOCAN 2012, Int. J. Cancer, 2015, 136, E359-E386.

2 S. Ruan, L. Zhang, J. Chen, T. Cao, Y. Yang, Y. Liu, Q. He, F. Gao and H. Gao, Targeting delivery and deep penetration using multistage nanoparticles for triplenegative breast cancer, RSC Adv., 2015, 5, 64303-64317.

3 K. F. Lei, B.-Y. Lin and N.-M. Tsang, Real-time and label-free impedimetric analysis of the formation and drug testing of tumor spheroids formed via the liquid overlay technique, RSC Adv., 2017, 7, 13939-13946.

4 F. L. Miles, F. L. Pruitt, K. L. van Golen and C. R. Cooper, Stepping out of the flow: capillary extravasation in cancer metastasis, Clin. Exp. Metastasis, 2008, 25, 305-324.

$5 \mathrm{P}$. Friedl and S. Alexander, Cancer invasion and the microenvironment: plasticity and reciprocity, Cell, 2011, 147, 992-1009.

6 F. Mbeunkui and D. J. Johann Jr, Cancer and the tumor microenvironment: a review of an essential relationship, Cancer Chemother. Pharmacol., 2009, 63, 571-582.

7 R. S. Watnick, The role of the tumor microenvironment in regulating angiogenesis, Cold Spring Harbor Perspect. Med., 2012, 2, a006676.

8 H. F. Dvorak, Tumor Stroma, Tumor Blood Vessels, and Antiangiogenesis Therapy, Cancer J., 2015, 21, 237-243.

9 N. Nishida, H. Yano, T. Nishida, T. Kamura and M. Kojiro, Angiogenesis in cancer, Vasc. Health Risk Manage., 2006, 2, 213-219.

10 D. Hanahan and R. A. Weinberg, Hallmarks of cancer: the next generation, Cell, 2011, 144, 646-674. 
11 K. M. Gray and K. M. Stroka, Vascular endothelial cell mechanosensing: new insights gained from biomimetic microfluidic models, Semin. Cell Dev. Biol., 2017, 71, 106117.

12 H. Yamaguchi, F. Pixley and J. Condeelis, Invadopodia and podosomes in tumor invasion, Eur. J. Cell Biol., 2006, 85, 213-218.

13 N. Peela, D. Truong, H. Saini, H. Chu, S. Mashaghi, S. L. Ham, S. Singh, H. Tavana, B. Mosadegh and M. Nikkhah, Advanced biomaterials and microengineering technologies to recapitulate the stepwise process of cancer metastasis, Biomaterials, 2017, 133, 176-207.

14 K. H. Benam, S. Dauth, B. Hassell, A. Herland, A. Jain, K. J. Jang, K. Karalis, H. J. Kim, L. MacQueen, R. Mahmoodian, S. Musah, Y. S. Torisawa, A. D. van der Meer, R. Villenave, M. Yadid, K. K. Parker and D. E. Ingber, Engineered in vitro disease models, Annu. Rev. Phytopathol., 2015, 10, 195-262.

15 D. Truong, J. Puleo, A. Llave, G. Mouneimne, R. D. Kamm and M. Nikkhah, Breast Cancer Cell Invasion into a Three Dimensional Tumor-Stroma Microenvironment, Sci. Rep., 2016, 6, 34094.

16 J. J. F. Sleeboom, J. Toonder and C. M. Sahlgren, MDA-MB231 Breast Cancer Cells and Their CSC Population Migrate Towards Low Oxygen in a Microfluidic Gradient Device, Int. J. Mol. Sci., 2018, 19, 3047.

17 I. K. Zervantonakis, S. K. Hughes-Alford, J. L. Charest, J. S. Condeelis, F. B. Gertler and R. D. Kamm, Threedimensional microfluidic model for tumor cell intravasation and endothelial barrier function, Proc. Natl. Acad. Sci. U. S. A., 2012, 109, 13515-13520.

18 H. F. Tsai, A. Trubelja, A. Q. Shen and G. Bao, Tumour-on-achip: microfluidic models of tumour morphology, growth and microenvironment, J. R. Soc., Interface, 2017, 14, 131.

19 D. Qin, Y. Xia and G. M. Whitesides, Soft lithography for micro- and nanoscale patterning, Nat. Protoc., 2010, 5, 491-502.

20 D. Gao, H. Liu, J. M. Lin, Y. Wang and Y. Jiang, Characterization of drug permeability in Caco-2 monolayers by mass spectrometry on a membrane-based microfluidic device, Lab Chip, 2013, 13, 978-985.

21 D. Huh, B. D. Matthews, A. Mammoto, M. Montoya-Zavala, H. Y. Hsin and D. E. Ingber, Reconstituting organ-level lung functions on a chip, Science, 2010, 328, 1662-1668.

22 F. An, Y. Qu, Y. Luo, N. Fang, Y. Liu, Z. Gao, W. Zhao and B. Lin, A Laminated Microfluidic Device for Comprehensive Preclinical Testing in the Drug ADME Process, Sci. Rep., 2016, 6, 25022.

23 K. C. Chaw, M. Manimaran, E. H. Tay and S. Swaminathan, Multi-step microfluidic device for studying cancer metastasis, Lab Chip, 2007, 7, 1041-1047.

24 A. B. Al-Mehdi, K. Tozawa, A. B. Fisher, L. Shientag, A. Lee and R. J. Muschel, Intravascular origin of metastasis from the proliferation of endothelium-attached tumor cells: a new model for metastasis, Nat. Med., 2000, 6, 100-102.

25 V. L. Cross, Y. Zheng, N. Won Choi, S. S. Verbridge, B. A. Sutermaster, L. J. Bonassar, C. Fischbach and
A. D. Stroock, Dense type I collagen matrices that support cellular remodeling and microfabrication for studies of tumor angiogenesis and vasculogenesis in vitro, Biomaterials, 2010, 31, 8596-8607.

26 M. Moya, D. Tran and S. C. George, An integrated in vitro model of perfused tumor and cardiac tissue, Stem Cell Res. Ther., 2013, 4(suppl. 1), S15.

27 S. M. Albelda, P. M. Sampson, F. R. Haselton, J. M. McNiff, S. N. Mueller, S. K. Williams, A. P. Fishman and E. M. Levine, Permeability characteristics of cultured endothelial cell monolayers, J. Appl. Physiol., 1988, 64, 308322.

28 K. M. Chrobak, D. R. Potter and J. Tien, Formation of perfused, functional microvascular tubes in vitro, Microvasc. Res., 2006, 71, 185-196.

29 A. Ozcelikkale, H. R. Moon, M. Linnes and B. Han, In vitro microfluidic models of tumor microenvironment to screen transport of drugs and nanoparticles, Wiley Interdiscip. Rev.: Nanomed. Nanobiotechnol., 2017, 9, e1460.

30 T. A. Nguyen, T. I. Yin, D. Reyes and G. A. Urban, Microfluidic chip with integrated electrical cell-impedance sensing for monitoring single cancer cell migration in three-dimensional matrixes, Anal. Chem., 2013, 85, 1106811076.

31 Y. Choi, E. Hyun, J. Seo, C. Blundell, H. C. Kim, E. Lee, S. H. Lee, A. Moon, W. K. Moon and D. Huh, A microengineered pathophysiological model of early-stage breast cancer, Lab Chip, 2015, 15, 3350-3357.

32 C. L. Chaffer and R. A. Weinberg, A perspective on cancer cell metastasis, Science, 2011, 331, 1559-1564.

33 N. Peela, F. S. Sam, W. Christenson, D. Truong, A. W. Watson, G. Mouneimne, R. Ros and M. Nikkhah, A three dimensional micropatterned tumor model for breast cancer cell migration studies, Biomaterials, 2016, 81, 72-83.

34 A. C. Shieh and M. A. Swartz, Regulation of tumor invasion by interstitial fluid flow, Phys. Biol., 2011, 8, 015012.

35 J. D. Shields, M. E. Fleury, C. Yong, A. A. Tomei, G. J. Randolph and M. A. Swartz, Autologous chemotaxis as a mechanism of tumor cell homing to lymphatics via interstitial flow and autocrine CCR7 signaling, Cancer Cell, 2007, 11, 526-538.

36 M. E. Fleury, K. C. Boardman and M. A. Swartz, Autologous morphogen gradients by subtle interstitial flow and matrix interactions, Biophys. J., 2006, 91, 113-121.

37 N. Reymond, B. B. d'Agua and A. J. Ridley, Crossing the endothelial barrier during metastasis, Nat. Rev. Cancer, 2013, 13, 858-870.

38 S. P. Chiang, R. M. Cabrera and J. E. Segall, Tumor cell intravasation, Am. J. Physiol.: Cell Physiol., 2016, 311, C1C14.

39 X. Y. Wang, Y. Pei, M. Xie, Z. H. Jin, Y. S. Xiao, Y. Wang, L. N. Zhang, Y. Li and W. H. Huang, An artificial blood vessel implanted three-dimensional microsystem for modeling transvascular migration of tumor cells, Lab Chip, 2015, 15, 1178-1187.

40 C. Zheng, L. Zhao, G. Chen, Y. Zhou, Y. Pang and Y. Huang, Quantitative study of the dynamic tumor-endothelial cell 
interactions through an integrated microfluidic coculture system, Anal. Chem., 2012, 84, 2088-2093.

$41 \mathrm{~N}$. Weidner, New paradigm for vessel intravasation by tumor cells, Am. J. Pathol., 2002, 160, 1937-1939.

42 P. Friedl and K. Wolf, Tumour-cell invasion and migration: diversity and escape mechanisms, Nat. Rev. Cancer, 2003, 3, 362-374.

43 M. Bockhorn, R. K. Jain and L. L. Munn, Active versus passive mechanisms in metastasis: do cancer cells crawl into vessels, or are they pushed?, Lancet Oncol., 2007, 8, 444-448.

44 J. W. Song, S. P. Cavnar, A. C. Walker, K. E. Luker, M. Gupta, Y. C. Tung, G. D. Luker and S. Takayama, Microfluidic endothelium for studying the intravascular adhesion of metastatic breast cancer cells, PLoS One, 2009, 4, e5756.

45 M. Arif, P. Kitchen, M. T. Conner, E. J. Hill, D. Nagel, R. M. Bill, S. J. Dunmore, A. L. Armesilla, S. Gross, A. R. Carmichael, A. C. Conner and J. E. Brown, Downregulation of aquaporin 3 inhibits cellular proliferation, migration and invasion in the MDA-MB-231 breast cancer cell line, Oncol. Lett., 2018, 16, 713-720.

46 C. Kim, J. Kasuya, J. Jeon, S. Chung and R. D. Kamm, A quantitative microfluidic angiogenesis screen for studying anti-angiogenic therapeutic drugs, Lab Chip, 2015, 15, 301310. 